\title{
Reducing the Frequent Switch Over between the Base Stations through Continuous Analysis of Signal Strength
}

\author{
M. R. Regitha ${ }^{1}$ and Varghese Paul ${ }^{2}$ \\ ${ }^{1}$ Department of Computer Science, Sacred Heart College, Kochi, Kerala, India \\ ${ }^{2}$ Department of Information Technology, Cochin University of Science and Technology, Kochi, Kerala, India \\ E-Mail: regitha.baiju@gmail.com
}

\begin{abstract}
As the increase in the number of mobiles and mobile networks, the existing standards and technologies face several issues and challenges. In the mobility management of cellular networks, HO is the important parameter on which these issues and challenges affect drastically. Several research papers have proposed many ideas and schemes to reduce $\mathrm{HO}$ latency. As the increase in the number new technologies and networks, the existing ideas are no longer supported efficiently. So a continuous analysis on handover (HO) procedure is required. Signal strength (SS) and quality of service $(\mathrm{QoS})$ are measured at particular time intervals in cellular networks. Results show that the highest value of signal strength has the best quality of service. In this paper, a continuous analysis on signal strength received by the mobile station (MS) is used to monitor the HO process to reduce the HO delay. In this analysis, the parameters user movement pattern, topological position, signal strength and time are used which will be helpful to forecast the HO in advance. Using HO forecast minimum number of frequent switch over between the base stations (BSs) reduces the HO latency in mobile networks. Keywords: Signal Strength, Handoff, Quality of Signal, Pingpong effect, Threshold, Hysteresis, Dwell Time
\end{abstract}

\section{INTRODUCTION}

The second and third generation mobile systems works on radio spectrum. The difference in bandwidth and coverage area encourages the multi-network interface devices which have the capability to access the services of various networks. The 4G technology device has this multi-network interface facility because of its higher bandwidth, large data rate and smoother and quick handover (HO). But 4G technology is not available in all the areas where the user travels. This situation causes the happening of $\mathrm{HO}$ between different network systems and different standards.

BS distributes the services to the MS. HO will occur when the mobile station (MS) moves from one base stations (BS) to another BS's coverage area. $\mathrm{HO}$ is a process of reconfiguring the MS, the wireless network and backbone wired network to support communication after an MS enters a different BS of the wireless network. Normally during the HO process, a few milliseconds of interrupt will happen. For smooth and seamless communication, this delay should be reduced. There are two categories of $\mathrm{HO}$ which are hard $\mathrm{HO}$ and soft $\mathrm{HO}$.

The figure 1 shows the HO process, in which the MS travelling from cell of BS1 to cell of BS2 while a call is forwarded to it. At the t0, the MS connects with BS1. At time $\mathrm{t} 1$, the HO is happening in which, the connection of MS from BS1 is switched over to BS2 without any interruption. The active call of MS is completed at the time of $\mathrm{t} 2$. Then at the time of $\mathrm{t} 3$, the MS is leaving from BS2.

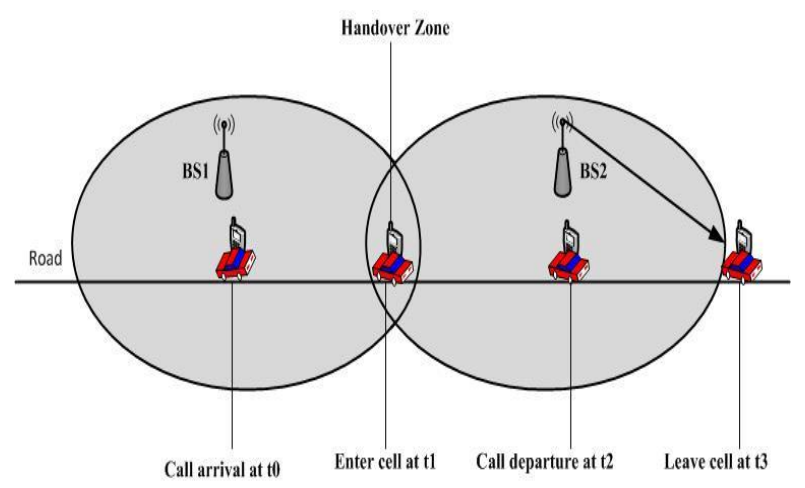

Fig. 1 Handover in Cellular Networks

The time interval in the complete $\mathrm{HO}$ process is known as $\mathrm{HO}$ latency. $\mathrm{HO}$ affects the quality of service directly. HO occurs if the signal quality falls below a predefined threshold level. The quality of service (QoS) and capacity of the network may be affected due to HO [3]. There are some requirements to reduce the adverse effect of $\mathrm{HO}$ such as $\mathrm{HO}$ latency must be as low as possible, the total number of $\mathrm{HO}$ should be minimal.

A hard HO means 'break before connect' which was used by the early systems. A mobile station can be connected only with one base station at a time. To solve this problem, MSC monitors the level of the signal for every few seconds. If the signal strength of the current cell becomes weak, MSC seeks a neighboring cell for better accommodation. MSC then changes the channel for carrying the call.

But soft HO means 'connect before break' which is used by the new systems. A mobile station can be connected with two base stations at a time. Before breaking the connection with the current base station, the mobile station can connect with the new cell during the HO. This causes a seamless connection. The network that uses GSM can be able to perform hard HO while the network which uses CDMA is able to perform soft $\mathrm{HO}$. 


\section{A. Cellular Network Architecture}

Cellular network which provides the communication between two moving units called MS or between one mobile unit and one stationary unit, called a land unit. A service provider locates and tracks a caller, assigns a channel to the call. As the caller travels, service provider transfers the channel from BS to another BS.

The figure 2 shows that each cellular service area is divided into small regions called cells. Each cell contains an antenna which is controlled a BS. Several BSs are controlled by a MSC which coordinates the communication between all the BSs and the computerized telephone central office. This centre is responsible for connecting calls, recording call information and creating billing.

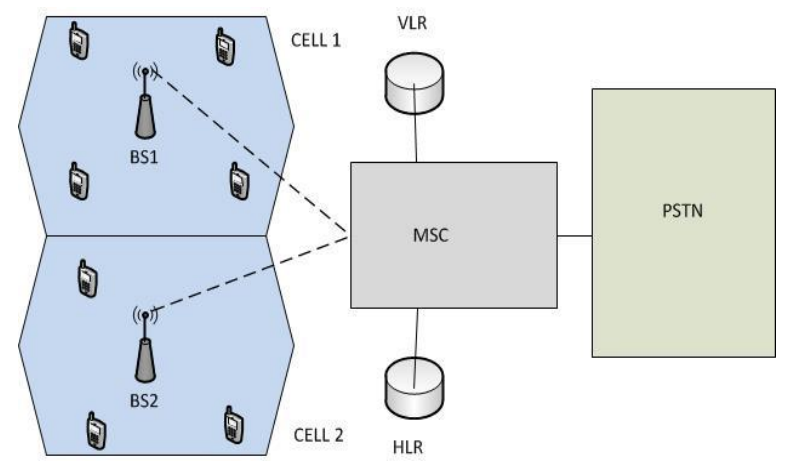

Fig. 2 GSM Cellular Network Architecture

\section{B. The Mobile Radio Channel Environment}

The mobile radio channel is a fading channel [3] at which the HO decision is used to detect the MS crossing the cell boundary. This can implemented by measuring RSS at MS from BS [1]. Due to multipath fading, the signal strength varies considerably. While an MS moving, the scatters and different terrain variations are happening which causes the rapid multipath fading by slow variation in the average signal pattern. The shadow fading follows log-normal distribution. Instead of a required single HO, multiple HOs (ping-pong) are happening due to the multipath and shadow fading [3]. These unnecessary HOs create problems in system. Firstly it increases the network load due to the requirement of resources by each $\mathrm{HO}$ and secondly causing shortage in channel resources leading to call dropping. To avoid such problems in the system, an efficient $\mathrm{HO}$ detection algorithm is necessary.

\section{Traditional Handoff Algorithm}

1. At every time strongest BS is selected in RSS based $\mathrm{HO}[2]$.

2. If the current signal is less than and target signal is stronger than the threshold (T), the HO is executed in RSS with threshold based HO.

3. If the current signal is less than and target signal is stronger than the hysteresis margin (n), the $\mathrm{HO}$ is occurred in RSS with hysteresis based HO. This type of HO prevents ping-pong effect [3][4].

4. If the current signal is less than the threshold and the target signal is stronger than the current one by a given hysteresis margin, the $\mathrm{HO}$ is executed in RSS with hysteresis and threshold based HO.

5. The following steps are added for improving the algorithm:

6. In cellular system wrong $\mathrm{HO}$ can be reduced by delaying the occurrence of $\mathrm{HO}$ until the target signal gets sufficiently stronger. To achieve this, absolute value of target signal strength is considered as threshold which has been involved in the signal strength based $\mathrm{HO}$ algorithm.

7. Proper setting with the new threshold value reduces the number of unnecessary HOs to the target BS when its signal strength is not sufficient to serve the call.

8. With the appropriate higher threshold value, the number of HOs occurring in the neighboring cells can be minimized.

\section{GSM Handoff Process: Problem faced}

Based on measurement data, hard handoff in GSM system creates a problem like unpredictable and highly irregular radio propagation in a real environment. The obstacles in a particular shadow fading produce some undesirable effects [4].

\section{Ping Pong Handoff}

The ping pong $\mathrm{HO}$ is a very undesirable effect that frequently occurs. In this scenario, due to power budget criterion, $\mathrm{HO}$ to a neighboring cell happens and returns to the original cell after a short time say less than 10 Seconds. Based on this criterion, a HO is mainly executed in cells with good radio coverage and minimal disruption due to interference. For avoiding such "ping-pong" effect, the MS is allowing to maintain a radio link with the current BS, until the signal strength from the target BS exceeds the signal strength of current BS by pre-calculated threshold value $\mathrm{H}$ some time called handoff Margin shown in figure 3. Besides the transmitting signal strength, the $\mathrm{HO}$ depends on the mobility of the MS. To prevent a minor variation in signal level of different BSs5, a threshold value of 5-10 dB is used in normal operation.

The large obstacles can cause strong shadow fading up to $30 \mathrm{~dB}$. If such a large obstacle is appeared in the line of sight of the current BS but not of the neighboring station, there is a possibility of a $\mathrm{HO}$ triggered. When the MS moves out of the shadowing area, the level of the signal strength again become normal and HO takes place to the current BS. The medium and high mobility of an MS, generates a $\mathrm{HO}$ to a neighboring $\mathrm{BS}$ and back to the current $\mathrm{BS}$ within a short period of time $<10 \mathrm{sec}$.

Handoff is only possible theoretically between points $a$ and $c$, but is recommended at point $\mathrm{b}$ because that is where the 
level of BS2 has first fallen below the allowable hysteresis value $h$.

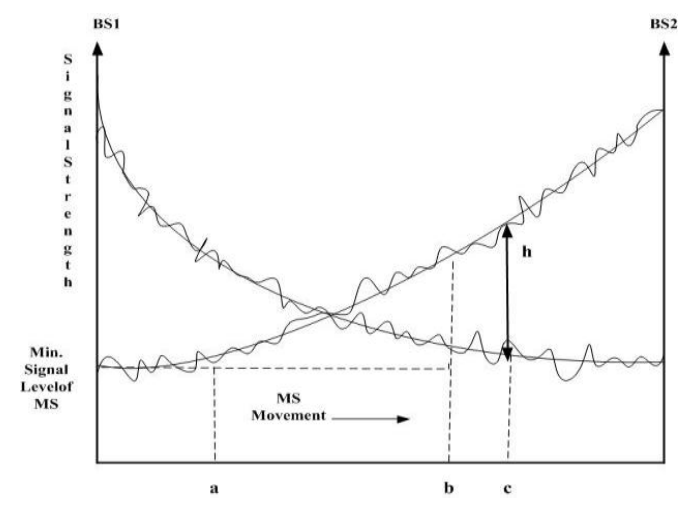

Fig. 3 GSM Handoff

\section{ANALYSIS OF MULTI CELL HANDOFF ALGORITHM}

A model has been developed for $\mathrm{HO}$ in two BSs in [5],[2],[6]. A model with three BSs is shown in figure 4. This model [7] has three base stations BS1, BS2, BS3 and a mobile station MS1 moving at a constant speed along the straight line path. The RSS at the MS from each of the three BSs are denoted by $\mathrm{RSS}_{\mathrm{bs} 1}, \mathrm{RSS}_{\mathrm{bs} 2}$, and $\mathrm{RSS}_{\mathrm{bs} 3}$ shown below.

The equation 1 to 3 is the sum of two terms, path loss and shadow fading. Rayleigh fading which also exists, but it can be neglected because it has much shorter distance when compared with shadow fading [8]. Rayleigh fading is attenuated by the low pass filter which is used for averaging but part of shadow fading is retaining. The RSS from three BSs expressed in $\mathrm{dB}$ can be written as [8].

$$
\begin{aligned}
& R S S_{b s 1}=\mu-\eta \log (d)+B S 1_{f d} \\
& R S S_{b s 2}=\mu-\eta \log (D-d)+B S 2_{f d} \\
& R S S_{b s 3}=\mu-\eta \log \sqrt{\left(\frac{D}{2}-d\right)^{2}+\left(\frac{3}{\sqrt{3}}-\frac{D}{2}\right)^{2}}+B S 3_{f d}
\end{aligned}
$$

Where,

1. D is the distance between two BSs.

2. $\mathrm{d}$ is the distance of the MS from BS2.

3. $\mu$ and $\eta$ are parameters for path loss.

4. $\mu$ depends on signal strength of BS.

5. $\eta$ is equivalent to path loss slope.

6. $\mathrm{RSS}_{\mathrm{fd} 1}, \mathrm{RSS}_{\mathrm{fd} 2}$ and $\mathrm{RSS}_{\mathrm{fd} 3}$ represent shadow fading

$\mathrm{BS} 1_{\mathrm{fd}}, \mathrm{BS} 2_{\mathrm{fd}}$ and $\mathrm{BS} 3_{\mathrm{fd}}$ indicate shadow fading, which follows log-normal distribution. Stationary Gaussian random processes in $\mathrm{dB}$ is represented by the zero in the log-normal distribution and assumed exponential, supported by measurement [9].

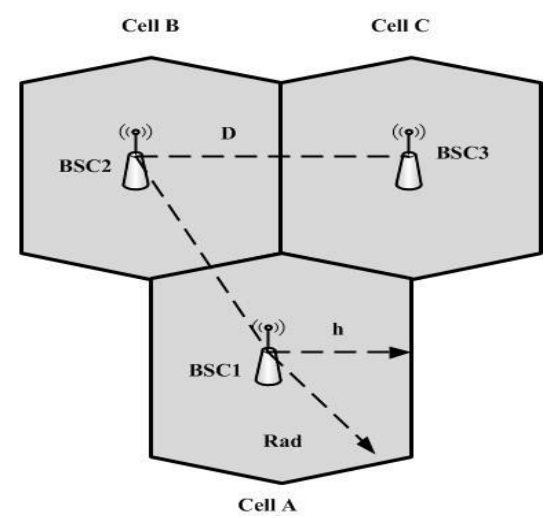

Fig. 4 Multi Cell Handoff

\section{SIMULATION}

The assumed values of parameters are simulated using NS2. The values are given below:

$\mu=0 \mathrm{~dB}, \eta=30, D=2000 \mathrm{~m}$, correlation distance $=20$, averaging constant $=30$ and standard deviation of the shadow fading $=6 \mathrm{~dB}$. By performing a test handoff is performed periodically at every sampling distance equal to $1 \mathrm{~m}$. The equations 1,2 and 3 are expressed as functions of distance so that the result is not dependent on the speed of the MS. The performance of the algorithm depends upon the number of handoffs, number of wrong handoff and expected average signal strength of the current BS to indicate delay.

In a mobile network, call initiation requests prioritize $\mathrm{HO}$ strategy. When an MS in a conversation moves from one BS to another BS, the HO is happening. HO transfers both the voice and control signals of an MS from a particular channel of a BS to another channel of another BS. Infrequent HOs must be performed successfully. A minimum usable signal level for acceptable voice quality $P \min$ is defined in HO procedure. Normal values of $P_{\min }$ are between $-85 \mathrm{dBm}$ and $-105 \mathrm{dBm}$. A stronger signal level $P_{t r h}$ is defined as threshold at which the HO is initiated. The MSC uses the differential $\Delta_{h_{\mathrm{nd}}}=P_{\mathrm{trh}}-P_{\min }$ to control HO procedure. The MSC is over engaged because too many HOs are occurred if $\Delta_{h_{\text {nd }}}$ is too large. Due to weak signal level, a call is lost because the insufficient time to complete a HO, if $\Delta_{h_{\text {nd }}}$ is too small.

The dwell time is the time over which a call may be maintained within a cell without HO. The propagation, interference, distance between the MS and BS are the measuring parameters to govern dwell time. In $1 \mathrm{G}$ and $2 \mathrm{G}$, the MSC supervises the $\mathrm{HO}$ while subsequent generations, $\mathrm{HO}$ is controlled by MS/BS. Enabling the transmission of the needed power to support a given data rate or sustaining a call in a mobile communication link is the main objective of optimal power control. Since higher transmitted power causes unnecessary interference but lower transmitted power increases the error rate which causes the call drop, or requires retransmission which invariably causes large transmission delays and lower throughputs. The quality of reception is determined by the received signal strength 
(RSS) of an MS from a BS. The RSS depends on the number of factors: quality of radio frequency planning, the number of BSs. The indoor radio propagation is difficult to predict the dense multipath environment and propagation effects such as reflection, diffraction, and scattering [10]. At particular locations, the multipath fading causes the received signal to fluctuate around a mean value. Usually the combined effects of large-scale fading and small-scale fading model the received signal. According to the inverse square law, an MS moves away from the serving BS, the RSS is dropping. According to the power law, the average RSS at any point decays as the square of the distance of separation between a transmitter and receiver. At a distance $\mathrm{d}$ from the transmitting antenna is calculated by the average received power $\mathrm{P}_{\text {avg }}$ [11].

$$
\begin{aligned}
& P_{a v g}=P_{s m l}\left(\frac{d}{d_{s m l}}\right)^{-n} \\
& P_{a v g}=P_{s m l}-10 n \log \left(\frac{d}{d_{s m l}}\right)
\end{aligned}
$$

where $P_{s m l}$ is received power at close-in reference point in the large coverage area of the BS at a small distance $d_{s m l}$ from the $\mathrm{BS}$, and $n$ is the path loss exponent. Using a developed App created, data was collected and arranged in the table I. From the collected data, a RSS-based localization of the network was computed. The computation involved the measurement of the RSS at different locations which meant to an estimated distance, $d$. To see the performance of the GSM network, the primary data obtained were analyzed graphically.

TABLE I RSS FOR A GSM NETWORK

\begin{tabular}{|c|c|c|c|c|}
\hline $\begin{array}{c}\text { RSS at MS } \\
(\mathbf{d B})\end{array}$ & $\begin{array}{c}\text { Mean RSS } \\
(\mathbf{d B})\end{array}$ & $\begin{array}{c}\text { Best RSS } \\
(\mathbf{d B})\end{array}$ & $\begin{array}{c}\text { RSS MD } \\
(\mathbf{d B})\end{array}$ & $\begin{array}{c}\text { RSS SD } \\
(\mathbf{d B})\end{array}$ \\
\hline-81 & -86.625 & -77 & 4.302083 & 31.15495 \\
\hline
\end{tabular}

\section{MATERIALS AND METHODS}

Using the App, the signal strength and quality of signals of a network is subjected to investigation and installed in Redmi 4 mobile phone. The Redmi 4 is connected to a computer system. The software automatically measures the signal strength of the BS in decibel micro volt per metre $(\mathrm{dBm})$. At 30 minutes intervals for 24 hours the RSS of MS is measured. Excel is used in generating graphs of the measured quantities against time to find out the lowest and the peak values and their effect on the network; while python and pandas are used in analysing the measured quantities. For simulation, the network simulator NS2 is used.

\section{RESULTS}

According to the figure 5, the signal strength (SS) of the network varied from $-77 \mathrm{dBm}$ and $-99 \mathrm{dBm}$ while the quality of signal (QoS) is varying from $58 \%$ and $12 \%$. The average SS $-79 \mathrm{dBm}$ at 7.00 hours and its corresponding average QoS of 56\%. A regression coefficient of 0.972 was found between its SS and QOS. The SS are recorded as stable along the period between the hours of 14:00 and 16:00.

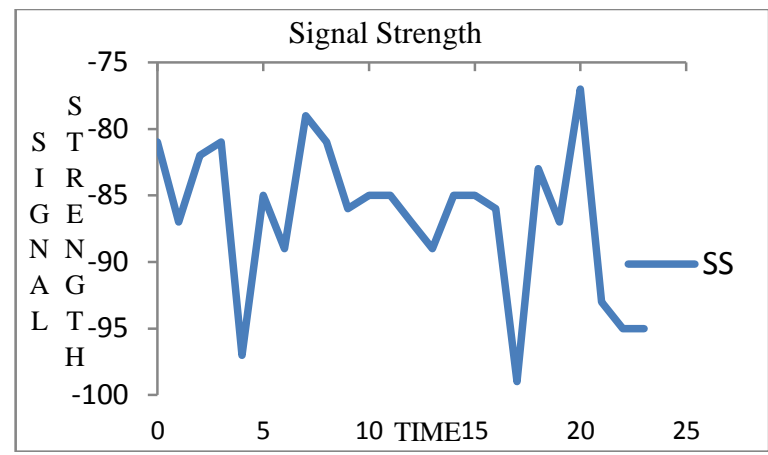

Fig. 5 Signal Strength Variation with Time for a GSM Network

\section{DISCUSSION}

The figure 5 shows the variation in signal strength which depicts that the technology used in the network does not influence on the signal strength. The signal strength is influenced by the distance from the transmitting BS and the obstacles but not by the technology used in the network [12]. There is no distinct variation in the signal strength with time in the day and at night. In the day time, the congestion does not affect the signal strength but it may affect data transmission within the network [13]. This paper is recommended that external antenna, signal booster and signal repeaters should be used to achieve a better signal strength in areas with poor signal strength. The signal strength is increased by these equipments tremendously [2]. However, to achieve a better signal strength in areas with poor signal strength, it is recommended that external antenna, signal booster and signal repeaters should be used. These equipments increase signal strengths tremendously [2].

\section{CONCLUSION}

This paper concludes that the signal strength is not affected by the technology used in the cellular networks. The factors affecting the signal strength are the distance from transmitting BS and obstacles. The signal strength is not influenced by the traffic congestion. This paper reveals that there is no distinction in signal strength between hours of heavy traffic and low traffic. The signal strength should be considered seriously since QoS provided by a network is a function of the signal strength of that network.

\section{REFERENCES}

[1] N. D. Tripathi, J. H. Reed and H. F, "Handoff in Cellular Systems", IEEE Pers. Commun., Vol. 5, No. 6, pp. 26-37, 1998, DOI: 10.1109/98.736475. 
[2] S. Saeed, C. Rezaei and B. H. Khalaj, "Grey Prediction Based Handoff Algorithm", International Scholarly and Scientific Research \& Innovation, Vol. 1, No. 2, 2007.

[3] S. Sheu, C. Wu and T. Hsien, "Using Grey Prediction Theory to Reduce Handoff Overhead in Cellular Communication Systems", in Proc. PIMRC, pp. 782-786, 2000, DOI: 10.1109/PIMRC.2000.881529.

[4] B. H. Walke, "Mobile Radio Networks: Networking, Protocols and Traffic Performance", 2nd ed., Wiley, pp. 992, 2002.

[5] P. Marichamy, S. Chakrabarti and S. L. Maskara, "Overview of Handoff Schemes in Cellular Mobile Networks and their Comparative Performance Evaluation", in Proc. VTC 1999-Fall, IEEE VTS 50th, Vol. 3, pp. 1486-1490, 1999, DOI: 10.1109/VETECF.1999.801509.

[6] N. Zhang and J. M. Holtzman, "Analysis of Handoff Algorithms using Both Absolute and Relative Measurements", IEEE Trans. Veh. Technol., Vol. 45, No. 1, pp. 174-179, 1996, DOI: 10.1109/25.481835.

[7] P. Marichamy, S. Chakrabarti and S. L. Maskara, "Performance Evaluation of Handoff Detection Schemes", in Proc. TENCON, Conf. Converg. Technol. Asia-Pacific Reg., Bangalore, India, pp. 643-646, 2003, DOI: 10.1109/TENCON.2003.1273250.

[8] S. A. Mawjoud, "Simulation of Handoff Techniques in Mobile Cellular Networks", Al-Rafidain Eng., Vol. 15, No. 4, pp. 31-39, 2007.
[9] M. Gudmundson, "Correlation Model for Shadow Fading in Mobile Radio Systems", Electron. Lett., Vol. 27, No. 23, pp. 2145-2146, 1991.

[10] M. Srbinovska, V. Dimcev, C. Gavrovski and Z. Kokolanski, "Localization Techniques in Wireless Sensor Networks using Measurement of Received Signal Strength Indicator", Electronics, Vol. 15, No. 1, pp. 67-71, 2011.

[11] N. Nkordeh, J. O. Olatunbosun, I. Bob-manuel, O. Oni and M. Iaeng, "Analysis of Mobile Networks Signal Strength for GSM Networks Analysis of Mobile Networks Signal Strength for GSM Networks", in Proc. WCECS, San Francisco, USA, pp. 19-21, 2016.

[12] E. C. Agbaraji, E. N. Gloria and O. Uzoma, "Cellular Mobile Signal Propagation; Effects of EIRP and Antenna Gain", J. Emerg. Trends Comput. Inf. Sci., Vol. 5, No. 3, pp. 172-177, 2014.

[13] P. A. K. Acharya, A. Sharma, E. M. Belding, K. C. Almeroth and K. Papagiannaki, "Congestion-aware rate adaptation in wireless networks: A Measurement-Driven Approach", in Proc. IEEE Commune. Soc. Conf. Sensor, Mesh Ad Hoc Commun. Networks, SECON, pp. 1-9, 2008, DOI: 10.1109/SAHCN.2008.11. 